\title{
Sufficient Conditions for Labelled 0-1 Laws
}

\author{
Stanley Burris ${ }^{1}$ and Karen Yeats ${ }^{2}$ \\ ${ }^{1}$ Department of Pure Mathematics, University of Waterloo, Waterloo, Ontario, N2L 3G1, Canada \\ ${ }^{2}$ Department of Mathematics and Statistics, Boston University, 111 Cummington St., Boston, MA 02215, USA
}

received December 12, 2006, revised February 2, 2008, accepted February 2, 2008.

If $\mathbf{F}(x)=e^{\mathbf{G}(x)}$, where $\mathbf{F}(x)=\sum f(n) x^{n}$ and $\mathbf{G}(x)=\sum g(n) x^{n}$, with $0 \leq g(n)=\mathrm{O}\left(n^{\theta n} / n !\right), \theta \in(0,1)$, and $\operatorname{gcd}(n: g(n)>0)=1$, then $f(n)=\mathrm{o}(f(n-1))$. This gives an answer to Compton's request in Question 8.3 [Compton 1987] for an "easily verifiable sufficient condition" to show that an adequate class of structures has a labelled first-order 0-1 law, namely it suffices to show that the labelled component count function is $\mathrm{O}\left(n^{\theta n}\right)$ for some $\theta \in(0,1)$. It also provides the means to recursively construct an adequate class of structures with a labelled 0-1 law but not an unlabelled 0-1 law, answering Compton's Question 8.4.

Keywords: ratio test, labelled structure, zero-one law

\section{Introduction}

Exponentiating a power series can have the effect of smoothing out the behavior of the coefficients. In this paper we look at conditions on the growth of the coefficients of $\mathbf{G}(x)=\sum g(n) x^{n}$, where $g(n) \geq 0$, which ensure that $f(n-1) / f(n) \rightarrow \infty$, where $\mathbf{F}(x)=e^{\mathbf{G}(x)}$. One application of this result is to 0-1 laws, where we find, see Theorem 7 , that if the labelled component count function for an adequate class of structures is $\mathrm{O}\left(n^{\theta n}\right)$ for some $\theta \in(0,1)$ then the class has a labelled monadic second-order 0-1 law.

Useful notation will be $f(n) \prec g(n)$ for $f(n)$ eventually less than $g(n)$ and $f(n) \in \mathrm{RT}_{\infty}$ for $f(n-1) / f(n) \rightarrow \infty$; the notation RT stands for the ratio test.

\section{The Coefficients of $e^{\text {poly }}$}

\section{Proposition 1 Given}

$$
\begin{aligned}
\mathbf{G}(x):= & g(1) x+\cdots+g(d) x^{d}, \quad g(i) \geq 0, g(d)>0, \\
& \text { with } \operatorname{gcd}(j \leq d: g(j)>0)=1 \\
\mathbf{F}(x):= & \sum_{n \geq 0} f(n) x^{n}=e^{\mathbf{G}(x)}
\end{aligned}
$$

the function $\mathbf{F}(x)$ is Hayman-admissible. Thus

$$
f(n) \sim \frac{\mathbf{F}\left(r_{n}\right)}{r_{n}^{n} \cdot \sqrt{2 \pi \mathbf{B}\left(r_{n}\right)}}
$$

1365-8050 @ 2008 Discrete Mathematics and Theoretical Computer Science (DMTCS), Nancy, France 
where $r_{n}$ is the unique positive solution to

$$
x \cdot \mathbf{G}^{\prime}(x)=n,
$$

and $\mathbf{B}(x):=x^{2} \mathbf{G}^{\prime \prime}(x)+x \mathbf{G}^{\prime}(x)$.

Proof: Theorem X of Hayman [5] shows that $\mathbf{F}(x)$ is Hayman-admissible. Then the rest of the claim is an immediate consequence of Corollary II of [5] where the saddle-point method is applied to find the asymptotics of the coefficients of an admissible function.

Corollary 2 For $\mathbf{F}(x), \mathbf{G}(x)$ as in the above proposition,

(a) $f(n) \in \mathrm{RT}_{\infty}$,

(b) $f(n)=\exp \left(-\frac{n \log n}{d}(1+\mathrm{o}(1))\right)$.

Proof: Itema follows immediately from Corollary IV of Hayman [5].

For item $\mathrm{b}$ one uses $r_{n} \mathbf{G}^{\prime}\left(r_{n}\right)=n$ to obtain:

$$
\begin{aligned}
\left(\frac{n}{c d g(d)}\right)^{1 / d} & \preceq r_{n} \leq\left(\frac{n}{d g(d)}\right)^{1 / d} \text { for } c>1 \\
r_{n} & =(1+\mathrm{o}(1))\left(\frac{n}{d g(d)}\right)^{1 / d} \\
r_{n}{ }^{n} & =(1+\mathrm{o}(1))^{n}\left(\frac{n}{d g(d)}\right)^{n / d} \\
\mathbf{B}\left(r_{n}\right) & =(1+\mathrm{o}(1)) d^{2} g(d)\left(\frac{n}{d g(d)}\right)=(1+\mathrm{o}(1)) d n \\
\mathbf{G}\left(r_{n}\right) & =(1+\mathrm{o}(1)) g(d) r_{n}{ }^{d}=(1+\mathrm{o}(1)) \frac{n}{d} \\
\mathbf{F}\left(r_{n}\right) & =\exp \left(\frac{n}{d}(1+\mathrm{o}(1))\right) .
\end{aligned}
$$

Apply these results to (1).

\section{Some Technical Lemmas}

Now we drop the assumption that $\mathbf{G}(x)$ is a polynomial, but keep the requirement

$$
\operatorname{gcd}(n: g(n)>0)=1 .
$$

This implies that $f(n) \succ 0$.

Choose a positive integer $L \geq 2$ sufficiently large so

$$
n>L \Rightarrow\left[x^{n}\right] \exp \left(g(1) x+\cdots+g(L) x^{L}\right)>0 .
$$


Given $\ell>L$ with $g(\ell)>0$ let

$$
\begin{aligned}
\mathbf{G}_{0}(x) & :=\sum_{n \geq 1} g_{0}(n) x^{n}:=\sum_{1 \leq n \leq \ell} g(n) x^{n} \\
\mathbf{F}_{0}(x) & :=\sum_{n \geq 0} f_{0}(n) x^{n}:=\exp \left(\mathbf{G}_{0}(x)\right) \\
\mathbf{G}_{1}(x) & :=\sum_{n \geq 1} g_{1}(n) x^{n}:=\sum_{n \geq \ell+1} g(n) x^{n} \\
\mathbf{F}_{1}(x) & :=\sum_{n \geq 0} f_{1}(n) x^{n}:=\exp \left(\mathbf{G}_{1}(x)\right) .
\end{aligned}
$$

Lemma 3 Suppose $r \geq-1$ is such that

$$
n g(n)=\mathrm{O}\left(f_{0}(n+r)\right)
$$

Then

$$
n f_{1}(n)=\mathrm{O}(f(n+r))
$$

Proof: In view of (3) and (5) we can choose $C_{r}$ such that

$$
n g(n) \leq C_{r} f_{0}(n+r) \text { for } n+r \geq L+1 .
$$

Differentiating (4) gives

$$
\begin{aligned}
n f_{1}(n) & =\sum_{j=\ell+1}^{n} j g(j) \cdot f_{1}(n-j) \\
& \leq C_{r} \sum_{j=\ell+1}^{n} f_{0}(j+r) \cdot f_{1}(n-j) \quad \text { by (6) } \\
& \leq C_{r} \sum_{j=0}^{n+r} f_{0}(j) \cdot f_{1}(n+r-j) \\
& =C_{r} f(n+r),
\end{aligned}
$$

the last line following from $\mathbf{F}(x)=\mathbf{F}_{0}(x) \cdot \mathbf{F}_{1}(x)$.

Lemma 4 Suppose for every integer $r \geq-1$

$$
n g(n)=\mathrm{O}\left(f_{0}(n+r)\right)
$$

Then $f(n-1) / f(n) \rightarrow \infty$.

Proof: Since $f_{0}(n) \in \mathrm{RT}_{\infty}$ by Corollary 2 there is a monotone decreasing function $\varepsilon(n)$ such that for any sufficiently large $M$ we have $\varepsilon(n)>f_{0}(n) / f_{0}(n-1)$ for $n \geq M$, and $\varepsilon(n) \rightarrow 0$ as $n \rightarrow \infty$. 
Thus

$$
\begin{aligned}
f(n) & =\sum_{0 \leq j \leq n} f_{0}(j) f_{1}(n-j) \\
& =\sum_{0 \leq j \leq M-1} f_{0}(j) f_{1}(n-j)+\sum_{M \leq j \leq n} f_{0}(j) f_{1}(n-j) \\
& \leq \mathrm{o}(f(n-1))+\varepsilon(M) \sum_{M \leq j \leq n} f_{0}(j-1) f_{1}(n-j)
\end{aligned}
$$

by Lemma 3 and the choice of $\varepsilon$

$$
\leq \mathrm{o}(f(n-1))+\varepsilon(M) f(n-1) \text {. }
$$

Thus

$$
\limsup _{n \rightarrow \infty} \frac{f(n)}{f(n-1)} \leq \varepsilon(M)
$$

and as $M$ can be arbitrarily large it follows that

$$
\lim _{n \rightarrow \infty} \frac{f(n)}{f(n-1)}=0
$$

\section{Main Result}

We are now in a position to prove the main result, making use of

$$
n !=\exp (n \log n \cdot(1+\mathrm{o}(1)))
$$

which follows from Stirling's result.

Theorem 5 Suppose $\mathbf{F}(x)=\exp (\mathbf{G}(x))$ with $\mathbf{F}(x)=\sum_{n \geq 0} f(n) x^{n}, \mathbf{G}(x)=\sum_{n \geq 1} g(n) x^{n}$, and $f(n), g(n) \geq 0$. Suppose also that $\operatorname{gcd}(n: g(n)>0)=1$ and that for some $\theta \in(0,1)$

$$
g(n)=\mathrm{O}\left(n^{\theta n} / n !\right)
$$

Then

$$
f(n) \in \mathrm{RT}_{\infty} .
$$

Proof: From Corollary 2, for any integer $r \geq-1$ and any $\theta \in(0,1)$, by choosing $\ell>L$ such that $1 / \ell<1-\theta$, we have

$$
\begin{aligned}
f_{0}(n+r) & =\exp \left(-\frac{(n+r) \log (n+r)}{\ell}(1+\mathrm{o}(1))\right) \\
& =\exp \left(-\frac{n \log n}{\ell}(1+\mathrm{o}(1))\right) \\
& \succ \frac{n^{\theta n}}{(n-1) !} .
\end{aligned}
$$

Thus $n g(n)=\mathrm{O}\left(f_{0}(n+r)\right)$. The Theorem then follows from Lemma 4 


\section{Best Possible Result}

The main result is in a natural sense the best possible.

Proposition 6 Suppose $t(n) \geq 0$ with $\operatorname{gcd}(n: t(n)>0)=1$ is such that for every $\theta \in(0,1)$

$$
t(n) \neq \mathrm{O}\left(n^{\theta n} / n !\right)
$$

Then there is a sequence $g(n) \geq 0$ with $\operatorname{gcd}(n: g(n)>0)=1$ and $g(n) \leq t(n)$ but $f(n) \notin \mathrm{RT}$, where one has $\mathbf{F}(x)=\exp (\mathbf{G}(x))$.

Proof: For $\theta \in(0,1)$ let

$$
S(\theta)=\left\{n \geq 1: t(n)>n^{\theta n} / n !\right\} .
$$

Then $S(\theta)$ is an infinite set.

Let $M$ be such that $\operatorname{gcd}(n \leq M: t(n)>0)=1$, and let

$$
\begin{aligned}
g_{1}(n) & := \begin{cases}t(n) & \text { if } n \leq M \\
0 & \text { if } n>M\end{cases} \\
\mathbf{G}_{1}(x) & :=\sum g_{1}(n) x^{n} \\
d_{1} & :=\operatorname{deg}\left(\mathbf{G}_{1}(x)\right) \\
\mathbf{F}_{1}(x) & :=e^{\mathbf{G}_{1}(x)} .
\end{aligned}
$$

For $m \geq 2$ we give a recursive procedure to define polynomials $\mathbf{G}_{m}(x)$; then letting

$$
\begin{aligned}
d_{m} & :=\operatorname{deg}\left(\mathbf{G}_{m}(x)\right) \\
\mathbf{F}_{m}(x) & :=e^{\mathbf{G}_{m}(x)}
\end{aligned}
$$

by Proposition 1

$$
f_{m}(n)=\exp \left(-\frac{n \log n}{d_{m}}(1+\mathrm{o}(1))\right) .
$$

To define $\mathbf{G}_{m+1}(x)$, having defined $\mathbf{G}_{m}(x)$, let

$$
h_{m}(n):=\frac{1}{n !} \cdot n^{\left(1-1 / 2 d_{m}\right) n} .
$$

Then

$$
\frac{h_{m}(n)}{f_{m}(n-1)} \rightarrow \infty \quad \text { as } n \rightarrow \infty .
$$

Thus we can choose an integer $d_{m+1}>d_{m}$ such that

$$
\begin{aligned}
d_{m+1} & \in S\left(1-\frac{1}{2 d_{m}}\right) \\
h_{m}\left(d_{m+1}\right) & >f_{m}\left(d_{m+1}-1\right) .
\end{aligned}
$$


This ensures that $h_{m}\left(d_{m+1}\right) \leq t\left(d_{m+1}\right)$. Let

$$
\mathbf{G}_{m+1}:=\mathbf{G}_{m}(x)+h_{m}\left(d_{m+1}\right) x^{d_{m+1}} .
$$

Then

$$
\frac{f_{m+1}\left(d_{m+1}\right)}{f_{m+1}\left(d_{m+1}-1\right)} \geq \frac{h_{m}\left(d_{m+1}\right)}{f_{m}\left(d_{m+1}-1\right)}>1
$$

Now let $\mathbf{G}(x)$ be the nonnegative power series defined by the sequence of polynomials $\mathbf{G}_{m}(x)$; and let $\mathbf{F}(x)=e^{\mathbf{G}(x)}$. Then $g(n) \leq t(n)$ but $f(n) \notin \mathrm{RT}_{\infty}$ as

$$
\frac{f\left(d_{m+1}\right)}{f\left(d_{m+1}-1\right)}=\frac{f_{m+1}\left(d_{m+1}\right)}{f_{m+1}\left(d_{m+1}-1\right)}>1 .
$$

\section{Application to $0-1$ laws}

A class $\mathcal{K}$ of finite relational structures is adequate if it is closed under disjoint union and the extraction of components. One can view the structures as being unlabelled with the component count function $p_{U}(n)$ and the total count function $a_{U}(n)$, both counting up to isomorphism. The corresponding ordinary generating series are

$$
\mathbf{P}_{U}(x):=\sum_{n \geq 1} p_{U}(n) x^{n}, \quad \mathbf{A}_{U}(x):=\sum_{n \geq 0} a_{U}(n) x^{n}
$$

connected by the fundamental equation

$$
\mathbf{A}_{U}(x)=\prod_{j \geq 1}\left(1-x^{j}\right)^{-p_{U}(j)} .
$$

One can also view the structures as being labelled (in all possible ways) with the count functions $p_{L}(n)$ for the connected members of $\mathcal{K}$, and $a_{L}(n)$ for all members of $\mathcal{K}$. The corresponding exponential generating series are

$$
\mathbf{P}_{L}(x):=\sum_{n \geq 1} p_{L}(n) x^{n} / n !, \quad \mathbf{A}_{L}(x):=\sum_{n \geq 0} a_{L}(n) x^{n} / n !
$$

connected by the fundamental equation

$$
\mathbf{A}_{L}(x)=e^{\mathbf{P}_{L}(x)} .
$$

All references to Compton in this section are to the two papers [3] and [4]. 


\subsection{Unlabelled 0-1 Laws for Adequate Classes}

Let $\mathcal{K}$ be an adequate class with unlabelled count functions and ordinary generating functions as described above. Compton showed that if the radius of convergence $\rho_{U}$ of $\mathbf{A}_{U}(x)$ is positive then $\mathcal{K}$ has an unlabelled 0-1 law ${ }^{(i)}$ iff $a_{U}(n) \in \mathrm{RT}_{1}$, that is,

$$
\frac{a_{U}(n-1)}{a_{U}(n)} \rightarrow 1 \text { as } n \rightarrow \infty
$$

$\mathcal{K}$ is finitely generated if $r=\sum p_{U}(n)<\infty$, that is, there are only finitely many connected structures in $\mathcal{K}$. In the finitely generated case the asymptotics for the coefficients $a_{U}(n)$ have long been known to have the simple polynomial form ${ }^{(i i)}$

$$
a_{U}(n) \sim C n^{r-1}
$$

provided $\operatorname{gcd}\left(n: p_{U}(n)>0\right)=1$. Item (9) leads to the fact that $a_{U}(n) \in \mathrm{RT}_{1}$, and hence to an unlabelled $0-1$ law. In addition to using this result, Compton notes that the work of Bateman and Erdös [1] shows that if $p_{U}(n) \in\{0,1\}$, for all $n$, then one has $a_{U}(n) \in \mathrm{RT}_{1}$.

Both of these results were subsumed in the powerful result of Bell [2] which says that if $p_{U}(n)$ is polynomially bounded, that is, there is a $c$ such that $p_{U}(n)=\mathrm{O}\left(n^{c}\right)$, then $a_{U}(n) \in \mathrm{RT}_{1}$.

\subsection{Labelled 0-1 Laws}

Compton shows that if $\rho_{L}$, the radius of convergence of $\mathbf{A}_{L}(x)$, is positive, then $\mathcal{K}$ has a labelled 0-1 law iff

$$
\frac{a_{L}(n-k) /(n-k) !}{a_{L}(n) / n !} \rightarrow \infty \quad \text { whenever } p_{L}(k)>0 .
$$

In particular it suffices to show that $a_{L}(n) / n ! \in \mathrm{R} \mathrm{T}_{\infty}$.

Compton's method to show that a given adequate class of finite relational structures $\mathcal{K}$ has a labelled 0 1 law is to show that its exponential generating function $\mathbf{A}_{L}(x)=\sum a_{L}(n) x^{n} / n$ ! is Hayman-admissible with an infinite radius of convergence. This guarantees that $a_{L}(n) / n ! \in \mathrm{RT}_{\infty}$ ([5], Corollary IV). However, as Compton notes, showing that $\mathbf{A}_{L}(x)$ is Hayman-admissible can be quite a challenge.

Question 8.3 of [3] first asks if, in the unlabelled case, the result of Bateman and Erdös, namely $p_{U}(n) \in$ $\{0,1\}$ implies $a_{U}(n) \in \mathrm{RT}_{1}$, can be extended to the much more general statement that $p_{U}(n)=\mathrm{O}\left(n^{k}\right)$ implies $a_{U}(n) \in \mathrm{RT}_{1}$, yielding an unlabelled $0-1$ law. As mentioned earlier, this was proved to be true by Bell. The second part of Question 8.3 asks if there is a simple sufficient condition along similar lines for the labelled case. We can now answer this in the affirmative with a result that is an excellent parallel to Bell's result for unlabelled structures.

Theorem 7 If $\mathcal{K}$ is an adequate class of structures with

$$
p_{L}(n)=\mathrm{O}\left(n^{\theta n}\right) \text { for some } \theta \in(0,1)
$$

then $a_{L}(n) / n ! \in \mathrm{RT} T_{\infty}$, and consequently $\mathcal{K}$ has a labelled monadic second-order $0-1$ law.

\footnotetext{
(i) Given a logic $\mathcal{L}, \mathcal{K}$ has an unlabelled $\mathcal{L} 0-1$ law means that for any $\mathcal{L}$ sentence $\varphi$, the probability that $\varphi$ holds in $\mathcal{K}$ will be either 0 or 1. In [3] Compton worked with first-order logic, in [4] with monadic second-order logic. In both papers he simply used the phrases "unlabeled 0-1 law" and "labeled 0-1 law".

(ii) This result is usually known as Schur's Theorem [6 3.15.2]. One can easily find the asymptotics 9] using a partial fraction decomposition of the right side of (7). The labelled case with finitely many components is more difficult-we needed to invoke Hayman's treatise [5] just to obtain the asymptotics for $\log a_{L}(n) / n$ ! (see Corollary2).
} 


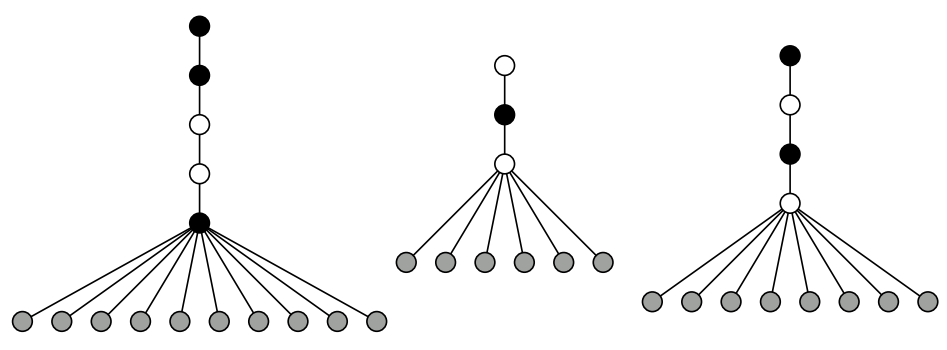

Fig. 1: Brooms with two-colored handles

Proof: This is an immediate consequence of Theorem 5 and Compton's proof that $a_{L}(n) / n ! \in \mathrm{R} \mathrm{T}_{\infty}$ guarantees such a $0-1$ law.

Now we list the examples of classes $\mathcal{K}$ which Compton shows have a labelled $0-1$ law, giving $p_{L}(n)$ in each case. It is trivial to check in each case that $p_{L}(n)=\mathrm{O}\left(n^{n / 2}\right)$; thus the $0-1$ law in each case follows from our Theorem 7 .

(a) 7.1 Unary Predicates $p_{L}(n)=0$ for $n>1$.

(b) 7.12 Forests of Rooted Trees of Height $1 \quad p_{L}(n)=n$.

(c) 7.15 Only Finitely Many Components $p_{L}(n)$ is eventually 0 .

(d) 7.16 Equivalence Relations $p_{L}(n)=1$.

(e) 7.17 Partitions with a Selection Subset $p_{L}(n)=2^{n}-1$.

We can now augment this list by, in each case, coloring the members of $\mathcal{K}$ by a fixed set of $r$ colors in all possible ways. This will increase the original $p_{L}(n)$ by a factor of at most $r^{n}$. This will still give $p_{L}(n)=\mathrm{O}\left(n^{n / 2}\right)$. Furthermore, in each of these colored cases let $\mathcal{P}$ be any subset of the connected members, and let $\mathcal{K}$ be the closure of $\mathcal{P}$ under disjoint union. Each such $\mathcal{K}$ has a labelled $0-1$ law.

Another application of Theorem 7 is to answer Question 4 of [3] by exhibiting an adequate class $\mathcal{K}$ such that $p_{L}(n)=\mathrm{O}\left(n^{3 n / 4}\right)$, hence there is a labelled $0-1$ law for $\mathcal{K}$; but also such that $\rho_{U} \in(0,1)$, so $\mathcal{K}$ does not have an unlabelled $0-1$ law.

Let the components of $\mathcal{K}$ be the one-element tree $T_{1}$ along with rooted trees $T_{3 n}$ of size $3 n$ and height $n$ consisting of a chain $C_{n}$ of $n$ nodes, with an antichain $L_{2 n}$ of $2 n$ nodes (the leaves of the tree) below the least member of the chain; and the chain $C_{n}$ is two-colored while the remaining nodes are uncolored. One can visualize these as brooms with 2-colored handles, see Figure 1.

The number of unlabelled components is given by $p_{U}(1)=1, p_{U}(3 n)=2^{n}$. Thus the radius of convergence of the ordinary generating function of $\mathcal{K}$ is $\rho_{U}=\sqrt[3]{2}$. Since this is positive and not 1 it follows from Theorem 5.9(ii) of [3] that $\mathcal{K}$ does not have an unlabelled 0-1 law. 
For the number $p_{L}(3 n)$ of labelled components of size $3 n$ :

$$
\begin{aligned}
p_{L}(3 n) & \leq 2^{n}\left(\begin{array}{c}
3 n \\
n
\end{array}\right) n ! \\
& \leq 2^{n}(3 n)^{n} \exp (n \log n \cdot(1+\mathrm{o}(1))) \\
& =\exp (2 n \log n \cdot(1+\mathrm{o}(1))) \\
& =(3 n)^{(2 / 3)(3 n)(1+\mathrm{o}(1))} \\
& =\mathrm{O}\left((3 n)^{(3 / 4)(3 n)}\right) .
\end{aligned}
$$

Thus $p_{L}(n)=\mathrm{O}\left(n^{3 n / 4}\right)$, so $a_{L}(n) / n ! \in \mathrm{RT} \infty$ by Theorem 7 , showing that $\mathcal{K}$ has a labelled $0-1$ law. 


\section{References}

[1] Bateman, P. T.; Erdős, P. Monotonicity of partition functions. Mathematika 3 (1956), 1-14.

[2] Bell, Jason P. Sufficient conditions for zero-one laws. Trans. Amer. Math. Soc. 354 (2002), no. 2, 613-630.

[3] Compton, Kevin J. A logical approach to asymptotic combinatorics. I. First order properties. Adv. in Math. 65 (1987), no. 1, 65-96.

[4] Compton, Kevin J. A logical approach to asymptotic combinatorics. II. Monadic second-order properties. J. Combin. Theory Ser. A 50 (1989), no. 1, 110-131.

[5] Hayman, W. K. A generalisation of Stirling's formula. J. Reine Angew. Math. 196 (1956), 67-95.

[6] Wilf, Herbert S. generatingfunctionology. Second edition. Academic Press, Inc., Boston, MA, 1994. $\mathrm{x}+228 \mathrm{pp}$. 